\title{
Les professionnels des services aux défunts : compétences, savoirs, qualifications
}

\author{
Sandrine Caroly, Valérie Rocchi, \\ Pascale Trompette et Dominique Vinck*
}

Fossoyeurs, toiletteur(se)s, porteurs, régleurs, maîtres de cérémonie... Les métiers funéraires sont d'anciens métiers qui ont traversé les siècles, portés par de multiples figures sociales : rôle social dévolu par la coutume, prolongement de la mission de soignant ou de religieux, emploi d'agent communal, spécialisation professionnelle au sein d'une entreprise. Avec la croissance des entreprises de pompes funèbres au $\mathrm{XX}^{\mathrm{e}}$ siècle et la marchandisation des services funéraires, ce dernier costume du « professionnel de service » a acquis une place dominante. Le vaste mouvement de médicalisation de la fin de vie a paradoxalement consacré une privatisation croissante de la prise en charge des défunts, avec la délégation du traitement du cadavre à ces spécialistes professionnels que sont devenues les pompes funèbres, selon une frontière symbolique relativement marquée entre champ de la santé et champ des services funéraires. La libéralisation du secteur en 1993, indissociable de l'établissement des pompes funèbres comme « profession réglementée », consacre ainsi l'avènement de ce groupe professionnel dans le contrôle du champ des services au défunt.

Partir aujourd'hui à la découverte de ces professionnels qui assurent la prise en charge des défunts, c'est donc rencontrer une communauté professionnelle relativement stabilisée dans la définition de son champ de compétence et du segment de marché du travail qui lui correspond. Mais c'est aussi, et dans le même temps, identifier la façon dont ce travail social continu de structuration professionnelle se poursuit et se prolonge aujourd'hui. La défense du territoire professionnel et la stabilisation de ses frontières (Abott, 1988), l'enrichissement du spectre des services et le développement de nouveaux champs de compétence, la construction de la qualification comme garantie de la qualité de la prestation, la conquête d'une légitimité sociale, etc., constituent autant d'enjeux qui animent aujourd'hui les mouvements de cette sphère professionnelle.

Nous proposons d'aborder ces dynamiques professionnelles en circulant d'un segment professionnel à un autre pour y travailler chaque fois une problématique spécifique. L'article se présentera ainsi comme une succession d'éclairages sur des lieux qui ont une portée heuristique pour l'analyse de l'évolution du champ des services au défunt.

\footnotetext{
* Sandrine Caroly : ergonome, maître de conférences, laboratoire CRISTO-UPMF Grenoble II. Valérie Rocchi, sociologue, chercheur contractuel, CRISTO-UPMF Grenoble II. Pascale Trompette, sociologue, chargée de recherche, CRISTO-UPMF Grenoble II. Dominique Vinck, sociologue, professeur, CRISTO-UPMF Grenoble II.
} 
Nous évoluerons tout d'abord à l'amont de la chaîne de traitement des défunts, à l'interface entre établissements de soins et opérateurs funéraires. Ici cohabitent deux populations, dominées par les catégories d'exécution, les agents de chambre mortuaire et les employés de pompes funèbres. Avec $70 \%$ des décès en milieu hospitalier, les relations entre soignants et personnels funéraires sont quotidiennes et engagent d'importantes contraintes de coordination. Cette interface entre secteur public et secteur privé se situe au cœur d'enjeux économiques importants. Après des décennies de désaffection face au problème de la mortalité au sein des établissements de soins, le renouvellement de la politique hospitalière en matière de prise en charge des mourants (soins palliatifs) et des défunts (soins au défunt) fait resurgir la problématique complexe de la division du travail entre hôpital et équipements privés, offre publique et offre marchande, soignants et pompes funèbres dans la chaîne des soins au défunt.

Dans un second temps, nous partirons à la rencontre de ce personnage emblématique de la profession de pompes funèbres qu'est le « conseiller funéraire ». Elle nous donnera accès aux principaux registres de compétence des opérateurs funéraires, tout au long d'une prestation de service qui conjugue traitement du corps, service public et ritualité funéraire. Maître d'œuvre des obsèques, le conseiller se découvre historiquement comme une figure à géométrie variable, évoluant d'une fonction de personnage public, au cœur des réseaux notables et religieux de la communauté locale, à celle de conseiller technico-commercial, cependant de plus en plus investi d'un rôle « d'officiant » dans la production des funérailles comme bien culturel et symbolique.

À distance de cette figure générique du métier de pompes funèbres, le thanatopracteur renvoie au contraire à une identité de spécialiste mobilisant des savoirs paramédicaux dans le traitement du cadavre. D'apparition relativement récente, ces « super-spécialistes » du traitement biologique du cadavre forment un segment professionnel qui cultive sa spécificité et son autonomie au sein de la famille des professionnels du funéraire. Il nous semble intéressant de nous attarder sur cette sous-communauté professionnelle, à la fois forte d'une position privilégiée et d'une légitimité supérieure dans la hiérarchie des métiers funéraires, et faible par la dispersion et l'hyperspécialisation des intervenants.

Tout au long de ces trois moments d'analyse, il s'agira d'être attentif à la façon dont ces mouvements renvoient à des déplacements dans le système des professions (Abott, 1988) attaché à ce champ de pratiques : comment s'établit ou se transforme la participation des différents groupes professionnels à la chaîne des soins au défunt ? Comment se (re)construit la division du travail entre les différents agents de traitement de la mort et quels en sont les enjeux ? Quelle redéfinition des relations et des échanges entre les différents segments professionnels ? Se dessine ici la problématique transversale de la structuration des territoires de compétences dans un champ 
interprofessionnel où cohabitent acteurs publics et privés, métiers de soins et métiers de conseil, spécialistes et maîtres d'œuvre, etc.

Nous disposons par ailleurs d'un important corpus de données sur la structure socio-économique du secteur et l'évolution de la réglementation, capitalisé à partir de précédentes recherches. Celles-ci étaient essentiellement consacrées à l'histoire du commerce funéraire et à la transformation du marché des services funéraires depuis la fin du monopole (Boissin, Trompette, 2002).

\section{Encadré 1 : Méthodologie générale de l'étude}

Cette étude a été réalisée dans le cadre du programme de recherche de la MiRe/DREES sur « Les dynamiques professionnelles dans le champ de la santé » et a donné lieu à un rapport : "Les services au défunt : acteurs, territoires de compétences et dynamiques professionnelles » (décembre 2003).

- La méthodologie d'enquête a principalement été basée sur l'analyse des situations de travail au sein des entreprises de pompes funèbres ainsi que des établissements hospitaliers. Le dispositif d'enquête a été conçu avec le souci de diversifier les contextes de situations de travail, notamment en terme de territoire (Paris, urbain, semi-rural, rural) et de structures d'entreprise pour les opérateurs funéraires (public/privé, grande entreprise/PME).

- Conjuguant entretiens semi-directifs et observations, le travail d'enquête s'est déployé en direction des trois principaux segments professionnels opérant dans le champ de la mort :

- les soignants, et principalement les personnels des chambres mortuaires (morgue), directement impliqués dans l'accueil et les premiers soins au défunt ;

- les thanatopracteurs, spécialisés dans le traitement du corps (toilette, soin, restauration), à la jonction entre monde médical et monde funéraire ;

- les pompes funèbres, associées à l'offre de services funéraires proprement dite (traitement administratif, hôtellerie funéraire, vente de produits, cérémonie, inhumation/crémation).

- Concernant le personnel hospitalier, le dispositif d'investigation s'est assorti d'une enquête par questionnaires, principalement réalisée au sein de l'Assistance publique des hôpitaux de Paris (AP-HP). Avec un taux de réponse de $15 \%$ sur une population cependant limitée en nombre (150), les résultats du questionnaire ont permis d'apporter des éléments d'analyse complémentaires sur le contexte de travail et l'activité des agents, l'évolution historique de la prise en charge hospitalière de la mort, les parcours professionnels et l'emploi, les relations interprofessionnelles.

La recherche s'est également appuyée sur des entretiens avec les nombreux représentants institutionnels de la profession, experts ou «praticiens réflexifs " porteurs d'une réflexion sur l'évolution des services au défunt :

- des cadres dirigeants des structures : cadres hospitaliers et responsables de chambres mortuaires, dirigeants de PME, cadres intermédiaires et fonctions spécialisées (recrutement, qualité, etc.) au sein de grandes entreprises ;

- d'autres intervenants professionnels tels que médecins, soignants en maison de retraite, membres du clergé, laïques responsables des cérémonies religieuses;

- des partenaires sociaux : syndicats salariés et fédérations patronales, intervenants au niveau de la formation professionnelle ;

- les « experts » : journalistes professionnels, consultants, intellectuels. 


\section{a Coordination et concurrence aux frontières : agents hospitaliers et employés funéraires}

Comprendre l'évolution des métiers du funéraire n'est possible qu'en se situant, dans un premier temps, en amont de la chaîne de traitement des défunts, à l'interface entre établissements de soins et opérateurs funéraires. En effet, le vaste mouvement de médicalisation de la fin de vie a paradoxalement consacré une privatisation croissante de la prise en charge des défunts, avec la délégation du traitement du cadavre à ces spécialistes professionnels que sont devenues les pompes funèbres, selon une frontière symbolique relativement marquée entre champ de la santé et champ des services funéraires.

Nous montrerons tout d'abord comment le traitement de la mort a été longtemps délaissé par le champ de la santé et explorerons ensuite la façon dont les professionnels des établissements de soins ont progressivement pris en charge les mourants, en relation aux nouvelles politiques hospitalières, conduisant à une division de travail plus complexe entre les agents hospitaliers et les employés funéraires.

\section{La mort aux portes des établissements de santé}

L'exclusion de la mort du champ de la santé est d'abord et avant tout une problématique sociale. Car, si l'on prend pour point de départ la géographie de la mortalité, les établissements de santé - hôpitaux, maisons de retraite - constituent aujourd'hui les principaux lieux de concentration du phénomène. Conséquence directe de la médicalisation croissante de la fin de vie, $70 \%$ environ des décès se produisent en milieu hospitalier contre $30 \%$ au domicile ${ }^{1}$. Pourtant, pour les établissements de santé, le problème de la gestion de la mort a souvent été résolu par simple démission ${ }^{2}$, avec un transfert de responsabilités sur les entreprises privées. Comment expliquer cette désaffection des établissements de soins ? Plusieurs motifs peuvent être à l'origine d'une telle absence de traitement des défunts : la non-reconnaissance, à l'hôpital, des services qui n'ont pas une vocation «pure» de santé, une

1 Historiquement, c'est au tournant des années cinquante que le rapport entre le nombre de décès à domicile et le nombre de décès en milieu hospitalier (hôpital, maison de retraite, hospice) s'inverse : en 1964, on compte encore $63,2 \%$ de décès au domicile contre $33,5 \%$ à l'hôpital, au plan national. En 1983, 66 \% des décès ont lieu en milieu hospitalier et 30,5\% au domicile (Barrau, 1987). Ce ratio s'est encore accentué aujourd'hui.

2 Le rapport Aubert sur le secteur funéraire (1985) fait état du problème des retours clandestins des morts au domicile (en ambulance), " souvent incités par les établissements d'hospitalisation pressés de se "débarrasser" des corps des défunts ". Le rapport interministériel de 1989 sur l'organisation funéraire française porte une réflexion très critique sur le problème de la gestion de la fin de vie à l'hôpital : absence de politique, transfert de responsabilité sur les entreprises privées, "ghettoïsation » des personnels de morgue. "La mission porte une appréciation de sévérité sur l'irréflexion et le laisser-faire des pouvoirs publics dans un domaine qui ne peut laisser indifférent ». 
qualité de prestation de service aux familles du patient encore peu développée, des investissements économiques faibles pour les activités médicales non techniques, un déni de la mort, une gestion des cadavres uniquement dans le cadre de l'autopsie dans des unités d'anatomo-pathologie, etc.

Les pompes funèbres ont longtemps pallié cette carence et ont investi dans les soins au défunt et dans les équipements tels que la chambre funéraire. Annexée à l'établissement de santé, la chambre funéraire participe immédiatement au processus de traitement des cadavres. La conservation en casier réfrigéré s'établit pratiquement et symboliquement comme une étape supplémentaire de la chaîne du soin ou du « postsoin ». Les employés funéraires évoluent librement au sein de l'hôpital pour la levée du corps, se substituent au soignant pour la toilette mortuaire, tandis que la chambre funéraire se confond avec la morgue, à défaut d'unité en propre consacrée à cet effet.

Plutôt que d'investir dans des équipements ad hoc, les hôpitaux ont largement profité de l'offre des opérateurs privés, une offre rapide, efficace et moins coûteuse ${ }^{1}$. L'absence de stricte réglementation en la matière, l'engouement des hôpitaux pour des solutions externes susceptibles de pallier rapidement le déficit d'équipement, l'intérêt pour les entreprises de pompes funèbres de se rapprocher de ces lieux de concentration de la mortalité, a encouragé le développement de morgues privées. Or, ce problème de carence hospitalière n'a fait qu'accroître une mauvaise prise en charge des mourants par le service hospitalier. La localisation de l'amphithéâtre (morgue) dans l'hôpital, par exemple, en dit long sur le rejet de cette activité post mortem : l'accès à la morgue se situe la plupart du temps à distance des unités de soins et coïncide parfois avec le stockage et l'évacuation des déchets. Les dénominations ("morgue », " dépôt», " caveau ») ainsi que le rattachement institutionnel (intendance, cuisine, logistique) marquent clairement une mise à distance et l'absence d'une politique de gestion de la fin de vie, conduisant à une « ghettoïsation » des personnels de la morgue.

" Longtemps la chambre mortuaire a été au bout de l'hôpital parce qu'on ne voulait pas mêler les morts et les vivants. Et donc souvent la chambre mortuaire est d'ailleurs à l'extérieur, à l'autre bout de l'hôpital avec une sortie sur une rue. C'est classique, on ne voit jamais un corbillard sortir de l'hôpital. Pour ces raisons, on a voulu toujours mettre les amphithéâtres assez loin, on a créé des lieux de solitude et d'ailleurs ils sont tous seuls souvent dans leur petit coin » (cadre hospitalier).

1 Pour l'opérateur funéraire, la chambre funéraire est avant tout un équipement de captation de la clientèle. L'avantage concurrentiel qu'il constitue lui permet aisément d'offrir des facilités, voire une gratuité de la prise en charge, en échange d'accords effectifs ou tacites avec les établissements de soins pour accroître les flux en direction de la chambre funéraire : l'hébergement temporaire gratuit, le transport gratuit, la rapidité d'intervention, la qualité de la coordination constituent autant d'arrangements pratiques et commerciaux dispensés par les opérateurs propriétaires ou gérants (par délégation) d'une chambre funéraire pour faciliter le transit direct de la chambre d'hôpital à la chambre funéraire. 


\section{Trajectoires professionnelles}

Le parcours professionnel des agents de chambre mortuaire peut prendre des formes diverses selon les choix d'itinéraires, les difficultés de recrutement, les types de formation reçue et souhaitée, les possibilités de gestion de carrière et l'identité au travail. Néanmoins, l'analyse détaillée de vingtcinq itinéraires d'agents de chambre mortuaire fait apparaître deux grands types de parcours : des agents issus du milieu hospitalier et des agents ayant réalisé une première carrière au sein du secteur des pompes funèbres. Quelle que soit l'origine, la plupart des agents ont connu ou ont côtoyé la chambre mortuaire par leurs anciens métiers, ce qui a facilité l'accès à la chambre mortuaire. Selon les deux grands types de parcours, diverses modalités d'entrée dans le métier sont possibles. Pour les agents ayant un parcours uniquement dans le milieu hospitalier, la plupart sont passés par plusieurs services (bloc, réanimation, chirurgie, soins intensifs, urgence, gériatrie) avant d'arriver à la chambre mortuaire. Les autres viennent d'un service technique (pour ces derniers le passage à la chambre mortuaire se marque par une augmentation de la charge émotionnelle dans la réalisation du travail). Enfin, dans les parcours «hôpital», beaucoup d'agents sont passés par une fonction de brancardier avant d'intégrer la chambre mortuaire. Pour les agents ayant eu d'abord un parcours par le secteur des pompes funèbres, l'entrée en chambre mortuaire se fait soit directement après avoir exercé un métier funéraire (souvent porteur) ou indirectement après avoir occupé un autre métier : pompier, relations de services (vendeur, sécurité) et parfois un passage par l'hôpital.

On constate que les motifs d'entrée dans le métier sont relativement variés. Pour quelques-uns, une personne de l'entourage a pu avoir une influence. Mais l'hétérogénéité reste importante. Une fois établi en chambre mortuaire, le personnel apprécie le cadre de travail du service public et ne cherche pas à développer des projets de mobilité. Les trajectoires s'arrêtent souvent en chambre mortuaire avec une volonté de valoriser leur compétence au sein de la structure hospitalière. Mais rester devient aussi un choix par défaut : on reste parce qu'il n'est pas facile de valoriser ses compétences sur le marché du travail.

Les agents hospitaliers directement affectés au traitement des défunts ont longtemps constitué un segment excessivement marginalisé et dévalorisé au sein des établissements de santé, situation encore très répandue aujourd'hui. Leur statut, fixé par le décret de 1991 et 1997, les situe au plus bas niveau de qualification, équivalent à n'importe quel agent des services techniques des établissements publics. Ils ont donc un horizon de carrière plus que limité, voir inexistant : " souvent c'est considéré comme le dernier des travails » (cadre hospitalier). Celui qui va travailler à la chambre mortuaire dans les hôpitaux est encore discriminé par rapport aux autres services de soins. Mais s'ils ont du mal à être reconnus par le monde des soignants, les agents d'amphithéâtre trouvent d'autres ressources 
communautaires dans l'interface avec les agents des pompes funèbres. Finalement, les identités professionnelles de ces agents publics ou privés tendent à se rejoindre dans une sorte de marginalité et de repli communautaire ${ }^{1}$. Autour des défunts, les frontières d'appartenance se recomposent, s'étoffent et s'enrichissent des affinités et des liens professionnels marqués entre les différents agents de la chaîne des soins au défunt. À observer les trajectoires professionnelles, on s'aperçoit que les frontières des organisations et des marchés spécifiques (public/privé) disparaissent, pour décrire un seul et même marché professionnel.

En 1989, une mission interministérielle ${ }^{2}$ en charge d'un rapport sur l'organisation des services funéraires en France fait un rapport très critique sur le problème de la gestion de la fin de vie à l'hôpital. Ce rapport conclut la nécessité de définir une véritable politique de gestion de la mort à l'hôpital, ce qui se concrétise à partir de 1997 par une réglementation imposant aux hôpitaux accueillant plus de 200 décès par an, l'obligation d'être équipés de chambres mortuaires avec des casiers réfrigérés et d'assurer l'accueil des familles du défunt (présentation du corps). En attendant, deux décennies de laisser-aller auront laissé des traces.

\section{Encadré 2 : Chambre funéraire et chambre mortuaire : une distinction entre offre privée et offre publique pour l'accueil des corps}

- Une chambre funéraire est une structure privée d'hébergement des corps dans l'attente d'une inhumation ou d'une crémation. Gérées par les opérateurs de pompes funèbres, les chambres funéraires participent à une offre de services concernant la conservation des corps, les soins au défunt et la présentation privée ou publique. La chambre funéraire s'associe à une gamme de services de qualité souvent supérieure à celle des morgues hospitalières, notamment pour la présentation en salon.

- La chambre mortuaire ou morgue hospitalière est destinée, comme la chambre funéraire, à recevoir avant inhumation ou crémation, le corps de personnes décédées. L'usage des chambres mortuaires est réservé au dépôt des corps des personnes décédées dans les établissements de santé. L'hébergement dans une chambre mortuaire est gratuit durant les trois jours qui suivent l'admission.

1 On peut notamment mentionner l'amicale des garçons de morgue au sein de l'AP-HP. Dans les sociétés de pompes funèbres, il existe des structures collectives plus ou moins formalisées.

2 En 1988, le ministre de l'Intérieur et le secrétaire d'État chargé des collectivités territoriales confient conjointement à l'inspection générale des Finances, l'inspection générale de l'Administration et à l'inspection générale des Affaires sociales, la mission d'analyser les insuffisances du régime juridique d'organisation des services funéraires en France et de proposer des orientations de réforme. 
Ces dernières années, la mort à l'hôpital n'est plus totalement considérée comme un échec de la mission médicale, mais comme une étape à accompagner dans le deuil des familles. En effet, la médicalisation de la fin de vie a produit un débat social autour des mourants. Avec la création des soins palliatifs, la fin de vie est devenue " objet d'intervention politique, mais d'une intervention confiée à une quasi-famille de professionnels » (Memmi, 2003). La réhabilitation des chambres mortuaires au sein des hôpitaux prolonge ce mouvement du côté de la prise en compte de l'immédiate après vie. Elle servira de tremplin à l'émergence d'un mouvement autonome de revendication des agents d'amphithéâtre. La transformation de la conception des chambres mortuaires a été portée avant tout par une poignée de militants engagés, au sein du monde des soignants (médecins, infirmiers, cadres hospitaliers, agents eux-mêmes), en particulier au sein de l'Assistance publique-Hôpitaux de Paris. La réhabilitation des agents mortuaires porte-t-elle en germe la remise en cause du monopole professionnel des pompes funèbres sur les soins aux défunts?

\section{La rébabilitation des chambres funéraires et la problématique de la division du travail des agents}

Il aura donc fallu la pression des pouvoirs publics pour que se mette en place une véritable politique de gestion de la mort à l'hôpital. Après des décennies de désaffection face au problème de la mortalité au sein des établissements de soins, le renouvellement de la politique hospitalière en matière de prise en charge des mourants (soins palliatifs) et des défunts (soins au défunt) fait resurgir la problématique complexe de la division du travail entre hôpital et équipements privés, offre publique et offre marchande, soignants et pompes funèbres dans la chaîne des soins au défunt. Cette interface entre secteur public et secteur privé se situe au cœur d'enjeux économiques importants ${ }^{1}$. Ici cohabitent deux populations, dominées par les catégories d'exécution, les agents de chambre mortuaire et les employés de pompes funèbres. Avec une grande majorité des décès en milieu hospitalier, les relations entre soignants et personnels funéraires sont quotidiennes et engagent d'importantes contraintes de coordination.

Rétablir la prise en charge du décès comme appartenant à la chaîne du soin, réaffilier les unités consacrées aux défunts au monde des soignants, renouer le fil coupé entre chambre mortuaire et monde médical, telle est l'ambition portée par un certain nombre de cadres hospitaliers. À partir du moment où le décès est rétabli comme objet à part entière de la mission hospitalière, les

1 On rappelle que l'hébergement dans une chambre mortuaire (amphithéâtre, morgue) est gratuit durant les trois jours qui suivent l'admission, tandis que l'hébergement dans une chambre funéraire est payant. 
identités professionnelles tendent à se recomposer, migrant d'une représentation proche de celle du «traitement des déchets » à celle d'une réelle fonction soignante.

L'apparition des soignants dans le champ des services aux défunts est relativement récente. Elle est longtemps demeurée suffisamment discrète pour ne pas menacer les professionnels qui occupaient le terrain. Avec la réhabilitation des soins au défunt au sein du monde hospitalier, les deux frontières des services mortuaires se trouvent interrogées : l'une, du côté des services cliniques, l'autre, du côté des opérateurs funéraires. Plusieurs hôpitaux évoluent vers une volonté de professionnaliser les agents d'amphithéâtre, avec l'embauche de personnels soignants. Les gestionnaires hospitaliers mobilisés par le renouvellement des populations des chambres mortuaires élaborent des stratégies pour faciliter le recrutement, avec des informations et des stages dans le cadre des formations dans les écoles de cadres ou dans les cours pour les aides-soignants. Dans le prolongement de cette orientation, ils tentent de recruter des femmes dans des métiers jusque-là réservés aux hommes, pour développer une attitude d'écoute et d'empathie des professionnels.

Les évolutions que nous venons de retracer, même si elles sont encore dépendantes de dynamiques locales propres à chacun des ensembles hospitaliers, sont porteuses de modifications substantielles dans le partage des territoires entre soignants et pompes funèbres. Il est maintenant intéressant de repérer quelques enjeux de ce déplacement sur la scène funéraire : comment cohabitent aujourd'hui les professionnels de la mort, au sein des chambres mortuaires?

La réflexion engagée sur la gestion de la mort en milieu de santé, la création des chambres mortuaires, l'affectation de nouveaux personnels, les réflexions en matière de formation, qualification et statut des agents de morgue, contribuent largement à une redéfinition de modes de division du travail antérieurs. Les interdépendances favorisent quelques arrangements en terme de disponibilités, de flexibilité des horaires d'ouverture, mais la coopération et la coordination restent souvent limitées. Prenons un exemple emblématique des problèmes de coordination entre les soignants, les agents d'amphithéâtre et les opérateurs funéraires : celui de la toilette du défunt. D'un côté, la toilette mortuaire relève de la compétence des infirmiers ${ }^{1}$ mais elle est très inégalement réalisée ou de façon relativement sommaire en vue de la présentation à la famille ${ }^{2}$. De l'autre, les agents mortuaires entrent pour la première fois dans le cadre de définition et d'apprentissage des compétences nécessaires à l'exercice de leur fonction. Enfin, pour les opérateurs funéraires, la toilette, et plus largement les soins au défunt, font

1 Elle est inscrite dans le décret de compétence des infirmiers, même si nombre d'entre eux s'en dispensent. Ceux qui demeurent soucieux d'assumer ce rôle sont généralement fortement exposés à la mort et le font au bénéfice de considérations éthiques très affirmées.

2 Elle ne comporte pas, notamment, la suture de bouche. 
pleinement partie de leur responsabilité et de leur champ professionnel et s'accompagnent d'un savoir sur les rites religieux ou les cultures traditionnelles du défunt. La toilette mortuaire fait donc l'objet d'une compétition entre les opérateurs privés et les agents mortuaires qui offrent gratuitement cette prestation aux familles. Au-delà des enjeux économiques, se joue ici la redéfinition des frontières des territoires de compétences. La coordination entre les professionnels est loin d'être évidente : "La réglementation prévoit qu'il y a une toilette faite par un infirmier, aide-soignant, et suivant les demandes des familles, soit le corps est habillé dans l'unité de soins, soit il est habillé dans la chambre mortuaire. En général, les agents mortuaires aiment bien le faire. Enfin, ils aiment bien... pas par goût, mais parce qu'ils estiment qu'ils savent mieux faire ". Il arrive souvent que la toilette soit exécutée plusieurs fois, au sein de l'établissement hospitalier puis par les pompes funèbres qui la factureront à la famille.

En l'absence de frontières strictes sur le partage des tâches, la problématique de la professionnalisation des agents mortuaires peut recouvrir des enjeux de développement des compétences pour les opérateurs funéraires. L'entraide semble alors difficile à organiser, ainsi que la coordination plus officieuse. L'enjeu de réhabilitation des soins aux défunts au sein du monde hospitalier est aussi celui de la reconquête de territoires jusqu'à présent dominés par les pompes funèbres. La concurrence entre chambre mortuaire et chambre funéraire porte également sur les soins au corps. Sur quels motifs repose l'interdiction aux soignants d'exercer ce domaine de compétences médicales, sinon celle du monopole professionnel des thanatopracteurs? La concurrence touche également l'exposition du corps, le cérémonial (chapelles ou espaces ouverts aux pratiques religieuses au sein de l'hôpital). La réhabilitation des chambres mortuaires à l'hôpital montre que la conquête du monopole du marché funéraire par les opérateurs privés n'est jamais définitivement acquise (Paradeise, 1987), mais soumise à des mouvements qui affectent le système des professions dans le champ des services aux défunts. Dans l'esprit des démarches de gestion de la qualité, l'hôpital s'inscrit aussi dans l'histoire de l'élaboration de référentiels de compétences et de l'amélioration des pratiques professionnelles. Seulement, cette qualité n'est pas conçue comme instrument d'innovation pour définir les frontières de compétences entre les opérateurs funéraires et les agents hospitaliers. Or, elle pourrait faire l'objet d'un dialogue sur les ajustements entre intervenants, sans toutefois s'imposer comme standard. Le point de départ pour comprendre cette qualité serait de décrire les pratiques professionnelles des uns et des autres, distinctes, éloignées, convergentes, redondantes, pour une coproduction du sens autour de l'accompagnement du défunt et une réelle activité distribuée. 


\section{Encadré 3 : La place du décès dans le champ de l'accréditation de l'ANAES}

Le manuel d'accréditation de l'ANAES, référentiel d'évaluation mis au point en 1999, est conçu comme un levier d'action important en vue de l'amélioration de la qualité du service rendu au sein des établissements de soins. L'accompagnement du décès n'apparaît pas comme une préoccupation centrale dans le cadre de cette démarche d'amélioration de la qualité.

De fait, quelques références du manuel y sont consacrées, concernant alternativement l'information et l'accompagnement du décès :

DIP. 5. g. L'entourage du patient est préalablement informé des autopsies (hors recherche légale des causes de décès).

Le décès du patient fait l'objet d'un accompagnement.

OPC. 13. a. Les personnes à prévenir sont contactées en cas d'état critique du patient.

OPC. 13. b. Les volontés et les convictions du défunt sont respectées.

OPC. 13. c. Le médecin traitant est informé du décès.

OPC. 13. d. Un accompagnement psychologique de l'entourage est assuré.

Cette simple mention permet cependant que la problématique du décès soit abordée par les auditeurs qui lui accordent nécessairement quelques attentions. En revanche, le manuel ne fait aucunement référence à la chambre mortuaire, à ses agents ou aux activités qui s'y déroulent. Le référentiel de l'ANAES, tout simplement, n'en dit rien. Le contraste est considérable avec le travail rendu public par M. Dupont et A. Macrez ${ }^{1}$ qui consacre près de cinquante pages au décès dans l'unité de soins et cent vingt pages à la chambre mortuaire, au transport du corps et aux opérations funéraires.

\section{- Les pompes funèbres... Vers la production de biens symboliques}

Parmi l'ensemble des personnels concourant à la transformation d'état du défunt, le conseiller funéraire (ou assistant funéraire) est certainement le plus important en raison de la position centrale qu'il occupe au sein du dispositif funéraire. Véritable maître d'œuvre des funérailles dès l'émergence des entreprises de pompes funèbres au $\mathrm{XIX}^{\mathrm{e}}$ siècle, ses compétences en la matière se sont considérablement enrichies depuis la seconde moitié de ce siècle et plus encore depuis 1993, date de l'ouverture du secteur à la concurrence. De pourvoyeur de fournitures mortuaires et coordonnateur des principaux acteurs funéraires (famille, représentants religieux et publics) à conseiller commercial et « prestataire » de biens symboliques, l'assistant funéraire voit son champ d'intervention s'élargir vers la dimension symbolique de la pratique funéraire.

1 Dupont M., Macrez A., Le décès à l'hôpital. Recommandations à l'usage des personnels, guides de l'APHP, Doin, 1998. 


\section{Encadré 4 : L’évolution de la législation funéraire : quelques repères}

- La loi du 28 décembre 1904, dans le cadre des dispositions relatives à la séparation de l'Église et de l'État, transfert aux communes, en matière de pompes funèbres, l'essentiel du contenu du monopole institué au profit des Églises un siècle plus tôt. Cette loi est intervenue en période anticléricale, juste avant la loi de séparation des Églises et de l'État du 9 décembre 1905 et, en ce qui concerne le secteur funéraire, dans la lignée des lois visant à assurer la neutralité de l'Administration et le respect de la liberté des funérailles.

- La loi du 9 janvier 1986 instaure un système de dérogation qui permet, dans certaines conditions, de faire échec au monopole exercé soit par la commune, soit par une entreprise de pompes funèbres agissant en concessionnaire. Cette loi s'avère être une première étape vers la suppression du monopole qui intervient en janvier 1993.

- La loi du 8 janvier 1993 abolit la notion de monopole dans le domaine funéraire et consacre la liberté pour les familles de choisir leur opérateur funéraire. Elle accroît la transparence du secteur funéraire au travers des dispositions du règlement national des pompes funèbres. Elle met en place les conditions d'une concurrence équilibrée et d'une garantie de qualité de service en instaurant un contrôle renforcé des qualités professionnelles des prestataires, sur la base de la procédure d'habilitation des opérateurs funéraires publics et privés. Elle fonde le Conseil national des opérations funéraires, organisme consultatif réunissant les représentants du secteur et ayant une mission de conseil des pouvoirs publics pour l'élaboration de la législation et de la réglementation funéraires.

- En application de la loi du 8 janvier 1993, le décret du 14 novembre 1997 précise un certain nombre d'obligations pour les établissements de santé publics et privés qui enregistrent un nombre moyen annuel de décès au moins égal à deux cents : d'une part, créer une chambre mortuaire et, d'autre part, mettre en place avant le 31 décembre 1998 un service mortuaire hospitalier qui devra accueillir les familles, assurer le dépôt et le séjour des corps pour lesquels le décret $n^{\circ}$ 94-1027 du 23 novembre prévoit la gratuité pendant trois jours. La création d'un service mortuaire conforme aux dispositions des textes mentionnés implique des dépenses d'investissements et d'exploitation importantes.

\section{Organiser la mise en scène des funérailles}

Le métier de conseiller funéraire est indissociable de la naissance des entreprises de pompes funèbres au début du XIX ${ }^{\mathrm{e}}$ siècle, avec le développement, au sein des grandes villes, d'entreprises spécialisées dans l'offre de prestations et fournitures nécessaires aux obsèques. Sous-traitantes auprès des institutions religieuses (fabriques et consistoires) et des municipalités détentrices du monopole public ou exclusivement pourvoyeuses de biens 
mortuaires «libres » ${ }^{1}$, ces entreprises ${ }^{2}$ se caractérisent par leur rôle d'organisatrices des funérailles et de pourvoyeuses de fournitures de décorum. Le conseiller funéraire se nomme ainsi " régleur » et exerce deux fonctions principales : la vente des fournitures et accessoires du convoi, à travers d'interminables listes de fournitures, dont certaines procurent des bénéfices substantiels à l'entreprise (fournitures libres); l'organisation proprement dite du convoi d'un point de vue logistique. À cette époque, les pompes funèbres signifient principalement l'ostentation des fastes et la théâtralisation de la mort. De fait, le régleur n'assiste pas aux funérailles, ce rôle étant dévolu à un agent spécialisé, le maître de cérémonie. Par ailleurs, le métier de régleur désigne une fonction spécifique au milieu urbain car dans le monde rural, la prise en charge du défunt reste du domaine de la sociabilité villageoise. Cela étant, il est un personnage public pris dans un réseau de relations locales très développé.

La coexistence entre les trois acteurs - publics, religieux et économiques est clairement délimitée sur des territoires d'activités relativement étanches en raison des régulations juridiques mises en place par Napoléon en 1804 et renforcées en 1905. Néanmoins, la mise en place du « service libre »- à côté du service intérieur réservé aux Églises et du service extérieur délégué aux entreprises mais fortement réglementé - crée une concurrence entre les fabriques et les entreprises privées sur la question des biens matériels. De fait, des tensions entre les deux acteurs sont visibles tout au long du $\mathrm{XIX}^{\mathrm{e}}$ siècle. La suppression du monopole des fabriques en 1905 redéfinit pour quelques dizaines d'années les territoires entre l'Institution religieuse catholique et les entreprises de pompes funèbres : à la première, la fourniture de biens symboliques, aux secondes la fourniture de biens matériels. De fait, à partir de cette époque, le régleur s'approprie l'ensemble des activités marchandes liées à la réalisation des convois (tentures, coussins...) et peaufine sa fonction au sein du dispositif funéraire.

\section{Gérer la transformation d'état : du cadavre au défunt}

La seconde moitié du $\mathrm{XX}^{\mathrm{e}}$ siècle voit se succéder toute une série de transformations sociales, économiques et juridiques qui ont des conséquences très importantes sur le métier de conseiller funéraire. Tout d'abord, le

\footnotetext{
1 On désigne ici l'ensemble des fournitures qui ne relèvent pas du champ du monopole tel que défini par la réglementation : fleurs, billets d'enterrement, ornements et accessoires en tout genre. La détermination des éléments constitutifs du monopole religieux puis communal donne lieu, de tout temps, à d'importantes controverses entre les différents protagonistes du commerce des services funéraires (municipalité, Eglise, entreprises). Elles sont résolues tantôt par la voie réglementaire, tantôt par la jurisprudence.

2 On distingue ici les sociétés adjudicataires du monopole et les « agences de funérailles » qui développent la concurrence à la périphérie du monopole. Tout au long du $\mathrm{XIX}^{\mathrm{e}}$ et $\mathrm{XX}^{\mathrm{e}}$ siècle, les sociétés privées concessionnaires du monopole communal chercheront à asseoir l'exclusivité de leur marché face au développement des agences de funérailles qui leur disputent la vente des fournitures libres.
} 
Concile Vatican II ${ }^{1}$ (1962-1965) institue d'une part un nouveau rituel des funérailles et d'autre part supprime la classe de convois et les fastes qui leur étaient afférents. Le rituel se veut sobre et dépouillé, la crémation est tolérée. En même temps, on constate un désinvestissement progressif de l'institution religieuse pour les funérailles qui ne sont pas un sacrement. Corollairement, un autre acteur émerge, caractérisé par une certaine ambiguïté : l'hôpital. À partir des années soixante, on l'a évoqué, le nombre de décès en milieu hospitalier augmente considérablement au point de devenir la norme, sans pour autant que les soignants n'investissent réellement le champ des services au défunt.

L'ensemble de ces évolutions a des conséquences directes sur les entreprises de pompes funèbres et de fait sur le métier de conseiller funéraire. Pour les premières, la fin du décorum mortuaire ouvre une nouvelle ère économique caractérisée par le développement de multiples services offerts aux familles, notamment dans le domaine du traitement du cadavre : conservation des corps dans des casiers réfrigérés, soins spécifiques tels ceux mis en œuvre par les thanatopracteurs, présentation du défunt dans des salons, cérémonies personnalisées - nous allons y revenir. L'entreprise de pompes funèbres devient alors véritablement l'acteur central du dispositif funéraire autour duquel les autres acteurs (public, soignant et religieux) se trouvent en quelque sorte satellisés. Cette position prédominante ne fera que se renforcer attirant d'ailleurs de nouveaux acteurs économiques totalement étrangers au secteur.

Dans ce contexte, le métier de conseiller funéraire s'est peu à peu enrichi de nouvelles compétences : relationnelles, organisationnelles et symboliques. Leur rôle central, que l'on peut qualifier de « maitre d'œuvre » dans la prise en charge du défunt, est particulièrement visible lors de l'entretien avec les familles. Cette prise en charge est totale et répartie dans une multitude de services, en sus de la fourniture de biens mortuaires (réception du cadavre, traitement et soin, conservation, organisation de la cérémonie, consultation téléphonique de l'acteur religieux, publications nécrologiques, hôtellerie funéraire, cartes de remerciements, etc.). L'interaction avec la famille nécessite de conjuguer des compétences commerciales (vendre des produits et des services à forte valeur ajoutée) et relationnelles liées aux capacités d'écoute, de compréhension, de gestion de l'émotion, de construction d'une relation de confiance (Caroly, Trompette, 2004). Cette double logique crée des tensions psychologiques parfois assez fortes, notamment dans le cas des décès d'enfants. Ces compétences s'élargissent en amont

1 L'enquête a porté sur la religion catholique uniquement. On estime à environ 70 à $80 \%$ le nombre d'enterrements catholiques actuellement. De plus, les pompes funèbres orientent leur «comportement» en fonction de l'Église catholique, les liens sont très forts et essentiels depuis au moins deux siècles. Les tensions qui se jouent entre le maître de cérémonie, le prêtre et les laïcs sont spécifiques et ne concernent pas les autres religions. Cela étant, ce sont ces tensions qui sont les plus importantes et les plus significatives de l'évolution de la profession de conseiller funéraire. 
avec le développement des contrats obsèques et en aval avec les services d'assistance au deuil. Les conseillers funéraires sont aujourd'hui très engagés dans la promotion et la vente des produits obsèques au point d'être parfois spécialisés au sein d'une agence. L'assistance au deuil ne fait pas partie directement de leur fonction mais la promotion de ces services, le plus souvent téléphoniques, relève de leur compétence. D'autres produits postfunérailles sont ainsi proposés tels que la prise en charge de toutes les démarches administratives consécutives au décès.

La réussite de cette relation de service particulièrement délicate nécessite, de la part du conseiller funéraire, d'autres compétences associées : les compétences d'agencement et de coordination des autres acteurs intervenants de manière plus ou moins directement dans la transformation d'état du défunt. La liste est longue : médecin, soignant, thanatopracteur, policier, employé de mairie, personnel religieux, opérateur gestionnaire d'un crématorium, etc. Il doit développer et cultiver un réseau au sein d'un territoire géographique important. L'activité de coordination se caractérise par des compétences d'ajustements en fonction de contraintes spécifiques et d'articulation entre des acteurs soumis également à de fortes contraintes (horaire et lieu de la cérémonie pour les personnels religieux). Enfin, la seconde compétence associée est liée au développement de la dimension symbolique comme produits et services à part entière fournis par les entreprises de pompes funèbres.

\section{Donner du sens : entre standardisation et authenticité}

L'ouverture à la concurrence, en 1993, a contraint les entreprises à redéfinir leurs prestations afin de se différencier (Vinck, 2003). Cela a donné lieu à la mise en place de démarches qualité, de standardisation des actes et des produits tout en proposant des combinaisons singulières selon les désirs et besoins des clients. Pour les entreprises, relève du symbolique tout ce qui n'est pas de l'ordre du matériel. Cela regroupe donc tout à la fois les comportements des personnels, la qualité des produits et matériels mis en œuvre ainsi que des prestations élaborées telles que les cérémonies et hommages. Chaque étape et chaque intervention des acteurs engagés dans le continuum de la transformation d'état sont décomposées en de multiples éléments qui sont ensuite analysés puis évalués et enfin intégrés ou rejetés. Au final, sont censées émerger des procédures standardisées auxquelles sont formés les personnels et particulièrement les conseillers funéraires en raison de leur position centrale.

Qu'il s'agisse de l'entretien avec la famille, de la mise en bière, de la fermeture du cercueil de la levée du corps, le conseiller funéraire se doit d'adopter des attitudes empreintes de respect, de dignité, de « distance chaleureuse ». Il s'agit également de veiller au bon déroulement des actions, selon une bonne mesure, ni trop lente, ni trop rapide, à la propreté des véhicules, à la tenue des porteurs, à la mise en place des fleurs, etc. L'accompagnement et 
le placement des familles sont une étape très importante lors de la cérémonie. Elle doit s'adapter au lieu, au public, au statut du défunt, au type de cérémonie, autant d'éléments clés auxquels doit s'adapter le conseiller funéraire. La diminution du nombre de pratiquants religieux et la montée en puissance des demandes de cérémonies personnalisées même au sein du cadre religieux, ont ouvert de nouvelles perspectives au conseiller funéraire : la direction des cérémonies et des hommages rendus au cimetière juste avant l'inhumation. Les entreprises ont créé de toutes pièces des petites cérémonies en agençant divers éléments symboliques empruntant le plus souvent à la symbolique chrétienne (la lumière, l'eau). Le conseiller funéraire se transforme alors en maître de cérémonie. Cette fonction, auparavant dévolue au porteur, est tenue soit par le chef d'agence soit par un conseiller funéraire expérimenté. Comme nous l'ont indiqué les personnels interrogés, elle est devenue « le top de la profession ».

Cela étant, il ne s'agit pas simplement d'une performance logistique ou comportementale. Le conseiller funéraire se pense comme un véritable médiateur symbolique. Il est censé réinjecter du sens où, selon lui, il n'y aurait plus que des actes mécaniques dans un contexte de désocialisation de la mort dans nos sociétés. Comment qualifie-t-il ce « sens » ? Il relèverait principalement de l'émotionnel et de l'authentique. Le conseiller funéraire cherche à introduire une charge affective dans chacun de ces actes et chacune des étapes de la transformation d'état du défunt. Cette dimension symbolique, telle qu'elle est rationalisée par les entreprises, s'appuie sur des valeurs et des manières de croire proprement modernes : individualisme, subjectivisme et relativisme permettent de composer des cérémonies singulières au sein desquelles le défunt est valorisé et où sa vie prend un sens à travers la mise en scène construite de toutes pièces par le conseiller funéraire. De fait, les entreprises de pompes funèbres contribuent, à l'instar d'autres acteurs à la catégorisation du mort et de la mort actuelle. Elles coproduisent, corégulent et diffusent des valeurs et des normes élaborées avec d'autres professions telles que les psychologues, les soignants, les religieux.

\section{Enjeux modernes du symbolique : les tensions interprofessionnelles}

Cette importance prise par les acteurs du funéraire dans le domaine symbolique suscite chez ses partenaires des réticences assez fortes. Sans entrer dans les détails, notons simplement que le conseiller funéraire, lorsqu'il endosse le costume de maître de cérémonie, se trouve face à deux autres types d'autorité symbolique : le prêtre et les laïcs. L'institution catholique $\mathrm{a}$, en effet, de grosses difficultés à assurer les enterrements et recourt très souvent aux laïcs et ce, d'autant plus que les lieux changent : ils peuvent faire des cérémonies dans des funérariums. Dans un contexte de sécularisation des sociétés occidentales et de demandes de cérémonies personnalisées, ces trois acteurs sont, d'une certaine manière, mis en concurrence. La 
dernière Pastorale des funérailles (1997) met en garde contre le développement des services offerts par les pompes funèbres en la matière qu'elle juge excessif et déplacé. L'Église cherche à restaurer son autorité et son contrôle non seulement sur les cérémonies mais également en amont et en aval du processus.

Si le siècle précédent a vu les deux acteurs s'affronter sur les questions des biens matériels, la fin du $\mathrm{XX}^{\mathrm{e}}$ siècle laisse entrevoir les tensions naissantes autour des biens symboliques, enjeu majeur dans une société faite de valeurs pluralistes et relativistes. Est-ce à dire que le conseiller funéraire deviendra, dans un avenir proche, un nouveau type d'autorité symbolique ? Dans le domaine spécifique des pratiques funéraires, il est possible que cette fonction se développe et capte une partie de la population qui souhaite laisser libre cours à son imagination pour leurs funérailles dans un cadre ni tout à fait religieux, ni tout à fait civil.

Ces cinquante dernières années ont été riches d'évolutions sociales, économiques et politiques qui ont durablement affecté le rapport au mort et à la mort. Le conseiller funéraire est devenu plus que jamais le maître d'œuvre du dispositif funéraire. Son rôle auprès des familles s'est renforcé et nécessite de nouvelles compétences sur des registres différents : techniques, relationnelles et symboliques. Ce dernier registre est sans doute le plus innovant d'autant plus qu'il n'en est encore qu'à ses balbutiements.

\section{- La « caste » des thanatopracteurs}

Si certaines spécialités de la pompe funèbre contemporaine abritent de très anciens métiers (fossoyeurs, porteurs, régleurs), celle qui engage le traitement du cadavre sur la base de techniques spécialisées de conservation, désignée de «thanatopraxie » en France, est d'apparition beaucoup plus récente. Elle apparaît au tournant du XIX ${ }^{\mathrm{e}}$ siècle, dans le sillage d'une série d'innovations affectant directement le domaine traditionnel des soins sur le corps du défunt. Nous nous intéresserons à la trajectoire singulière de ce groupe professionnel que sont les thanatopracteurs, tout au long d'un processus d'autonomisation qui en fait un segment à part entière au sein des professionnels des services au défunt, cependant pas totalement émancipé de sa tutelle originelle.

\section{La technicisation des soins au défunt}

De toutes les transformations qui affectent l'histoire du marché funéraire, l'extension de la gamme de services offerte par les pompes funèbres, notamment en matière de traitement du cadavre (transport, conservation, soin, présentation) est certainement la plus importante. Elle engage une modification pratique de l'activité, moins exclusivement concentrée sur 
l'habillage décoratif, et redéployée sur le traitement de la thanatomorphose et de l'ensemble des activités consécutives au décès. Elle traduit simultanément un déplacement dans la prise en charge professionnelle du cadavre, autrefois opérée au domicile par des femmes auxquelles la tradition dévouait ce rôle. Le traitement du corps, la présentation du défunt à l'entourage, le temps de la veillée ont en effet toujours constitué l'un des axes symboliques forts des funérailles dans leurs versions traditionnelles (Van Gennep, 1998) mais qui se déroulait au domicile du défunt. « Veilleuses », « pleureuses », « toiletteuses » - majoritairement des femmes - se chargeaient alors de l'apprêt du défunt avant les funérailles.

Avec la migration des mourants au sein des établissements de soins, tout se passe comme si la prise en charge médicale de la fin de vie se prolongeait par la prise en charge des morts par d'autres professionnels. Accompagnant ce mouvement d'appropriation du cadavre, les principales innovations portées par les pompes funèbres dans le contexte du développement de ce champ de compétences se concentrent sur le traitement du corps. Avec le développement des chambres funéraires, le cadavre a définitivement quitté le domicile pour reposer au sein d'un équipement spécialisé où interviennent des techniciens experts, susceptibles de prolonger la simple toilette par des procédés spécifiques de conservation du corps. La « thanatopraxie » est la dénomination française d'une technique pratiquée depuis longtemps au sein des pays anglo-saxons pour la conservation du cadavre sur la base de procédés d'embaumement (Lemonnier, 2004). Elle s'associe à la mise en œuvre de procédés d'asepsie, de désinfection et de restauration esthétique qui gomment les stigmates de la maladie et font obstacle au processus de décomposition ${ }^{1}$. La thanatopraxie fait aujourd'hui partie intégrante des services funéraires. Elle englobe l'ensemble des techniques mises en œuvre pour la conservation et la présentation des corps. Ces soins au défunt sont la plupart du temps facultatifs et visent à donner au visage un aspect naturel et à retarder le processus de décomposition.

\section{$D u$ « nettoyeur » au thanatopracteur}

On doit au groupe OFG-PFG, géant et leader national des pompes funèbres des années cinquante à aujourd'hui ${ }^{2}$, la paternité de ce « transfert technologique » des procédés de conservation depuis les États-Unis. Au début des

1 L'opération principale consiste en une injection intra-artérielle d'un produit antiseptique visant à retarder la décomposition, associée à l'extraction des gaz et liquides du corps. Cette opération facilite ainsi un éventuel transport du corps. La thanatopraxie peut également intégrer des opérations dites de « restauration », dans le cas d'accidents où les corps auraient été très abîmés, permettant ainsi de présenter ces derniers dans des conditions moins traumatisantes.

2 L'entreprise des Pompes funèbres générales a grandi tout au long du $\mathrm{XX}^{\mathrm{e}}$ siècle sur la base du développement et de la conquête du marché de la concession, qu'elle détient à $80 \%$ (2 900 communes) jusqu'à la libéralisation. À côté de ce leader d'envergure nationale (400 succursales réparties sur l'ensemble du territoire), la structure de la concurrence est atomisée. 
années soixante, OGF-PFG incite la petite société familiale Marette ${ }^{1}$, spécialisée dans l'activité de conservation par neige carbonique et désinfection et sous-traitant exclusif du groupe sur la région parisienne, à investir dans de nouvelles techniques d'embaumement. Francis Marette prospecte aux États-Unis. Il en rapporte une technique de traitement du cadavre rebaptisée en France thanatopraxie ou soins de conservation. Le procédé est déposé comme " procédé IFT », du nom de l'Institut français de thanatopraxie créé en 1963 et présidé par J. Marette - au sein duquel il est enseigné. En l'espace d'une décennie, la société Marette, rebaptisée Hygecobel, devient une grande entreprise de thanatopraxie ${ }^{2}$, filiale des PFG qui lui assurent l'exclusivité de la sous-traitance de l'activité de soins. Il faut attendre le milieu des années soixante-dix pour voir apparaître une première concurrence dans le domaine des soins, puis des équipements et produits d'aseptisation, avec l'entreprise Raffault ${ }^{3}$. Figure pionnière du développement de la thanatopraxie au côté de F. Marette, il développe son propre système de formation, en lien avec la faculté de Nice, en s'associant à l'institut EF2SM, école de formation de thanatopraxie fondée par la Fédération française de pompes funèbres, fédération des concurrents du groupe PFG.

De simples sous-traitantes des opérateurs funéraires, longtemps assignées à la partie la moins « noble » de la prestation, les entreprises de thanatopraxie se positionnent désormais comme de véritables expertes du traitement du cadavre. Leur développement n'en demeure pas moins annexé à la vaste entreprise de promotion des « soins de conservation » des opérateurs funéraires et au premier chef les PFG. En quelques décennies, la pratique des soins mortuaires, jusqu'alors réservée à la haute société, se démocratise et connaît un développement spectaculaire, au point d'être qualifiée d'évolution sociologique majeure (Thomas, 1980). «En France par exemple, en 1964, année où l'on commença à traiter les cadavres par le procédé de l'Institut français de thanatopraxie (IFT), on enregistra moins de 400 cas. En 1977, le nombre passa à 37061 pour atteindre 43496 en 1978, soit une augmentation de 17,36 \% au cours de ces deux années. En gros, il semble donc que le recours à la thanatopraxie concerne aujourd'hui $10 \%$ des décès

\footnotetext{
1 Créée en 1887 par Louis Marette, la société Marette est à l'origine une petite entreprise parisienne initialement spécialisée dans la teinturerie. En 1900, elle se tourne vers l'activité de désinfection de literies, locaux, vêtements après décès et connaît un développement important dans le contexte de la guerre de 1939-1945. Durant la période après-guerre, le fils de Louis Marette, Raymond, développe les procédés de conservation des corps par application de glace carbonique. L'entreprise exerce principalement en région parisienne, où elle ne rencontre que deux concurrents (Laleu à Saint-Ouen et Gilindre).

2 En 1968, Marette est une petite société d'une vingtaine de personnes. Les premières succursales s'ouvrent à Marseille et à Lille. En 1976, la société Marette se transforme en SA Hygecobel, dont le groupe OFG-PFG devient le principal actionnaire. À la même période, l'entreprise se diversifie du côté de la fabrication et de la vente de matériels et laboratoires post mortem destinés aux funérariums, hôpitaux et instituts de médecine légale. Ce sont là les débuts de la société Hygecobel qui fabrique et distribue aussi les instruments et produits de thanatopraxie. 3 Après être passé par l'entreprise Marette, C. Raffault fonde en 1974 sa propre entreprise, initialement spécialisée dans les toilettes et dans la conservation par application de neige carbonique. Il acquiert la maîtrise de la thanatopraxie sur la base de plusieurs stages réalisés en Angleterre et Belgique au terme desquels il obtient son diplôme de thanatopracteur.
} 
dans notre pays », observe L. V. Thomas (1980 : 128). Dix ans plus tard, le taux est passé à près de $20 \%$ (Barrau, 1987) soit plus de 100000 soins. Il est aujourd'hui de $37 \%$.

\section{L'établissement de juridictions professionnelles}

Le traitement du corps est devenu un nouveau champ de compétence à part entière des pompes funèbres. Les bénéfices de cette excroissance de leur champ de compétence sont loin de s'arrêter au développement d'une nouvelle offre de services. La problématique autour de laquelle s'élabore cette nouvelle professionnalité - le traitement du corps défunt - reconnaît des qualités tout à fait originales. Situées en dehors du champ couvert par la réglementation sur le service public des pompes funèbres, elles appellent très rapidement l'établissement de juridictions propres à offrir des garanties en matière d'hygiène, de police, de qualité de service. La question du « corps » engage bien d'autres enjeux que la simple fourniture des fastes. C'est effectivement autour de sa manipulation qu'apparaissent, à partir du début des années soixante-dix, un ensemble de juridictions destinées à encadrer les pratiques des opérateurs, notamment autour de la conformité technique des matériels (véhicule de transport) et des équipements (chambre funéraire), ainsi que du contrôle de l'activité.

L'activité de thanatopraxie hérite au premier chef de cette reprise en main des pouvoirs publics sur le développement tous azimuts de nouvelles pratiques en matière de traitement des défunts. Une série de juridictions ${ }^{1}$ participent à la production d'un premier cadre organisateur pour l'activité, incluant la normalisation des produits et la mise en place de procédures administratives minimales d'encadrement des pratiques ${ }^{2}$. Rien n'est encore dit des capacités professionnelles du praticien, que ce soit en termes d'obligation de formation ou de contrôle des qualités. Il faut attendre le vaste mouvement de réglementation du secteur, consécutif à la réforme de 1993, pour voir instauré le diplôme national de thanatopracteur ${ }^{3}$.

L'entrée des pompes funèbres dans le régime de la concurrence coïncide en effet avec le renforcement des dispositifs publics d'encadrement de l'activité ${ }^{4}$. Les thanatopracteurs sont doublement bénéficiaires des dispositions réglementaires prises dans ce contexte : d'une part, la détermination stricte

1 Décret no 76-435 du 18 mai 1976 et circulaire d'application du ministère de l'Intérieur du 5 juillet 1976.

2 Il s'agit notamment de l'autorisation de procéder aux soins de conservation, de l'homologation des fluides d'injection et de la simplification des modalités de contrôle de l'opération (Lemonnier, 2004).

3 Décret $\mathrm{n}^{\mathrm{o}}$ 94-260 du $1^{\mathrm{er}}$ avril 1994.

4 En définissant et fixant les prestations entrant dans le champ du « service extérieur des pompes funèbres » comme mission de service public, la loi de 1993 stabilise le territoire couvert par le monopole professionnel des pompes funèbres. Elle établit simultanément les juridictions propres à garantir un service de base de qualité (normalisation des équipements, produits, services) et le contrôle des qualités des professionnels habilités à exercer (habilitation). 
du champ d'intervention des professionnels habilités leur garantit l'absence de concurrence d'autres professions, notamment du côté de la vaste communauté des soignants auxquels l'exercice de l'activité est interdit ${ }^{1}$; d'autre part, les dispositions en matière de capacités professionnelles sont renforcées, les thanatopracteurs bénéficiant de la mise en place d'un diplôme d'État là où leurs prescripteurs (pompes funèbres) ne doivent satisfaire uniquement qu'à obligations de formation. Ce « supplément d'âme » est manifestement étroitement lié à leur affiliation avec le monde médical. Les pionniers de la thanatopraxie ont bâti leur système de formation en prenant pour pilier les médecins et les facultés de médecine. Ils ont développé des savoirs spécialisés en matière de traitement biologique du cadavre et de procédés techniques d'intervention. Cette stratégie de rapprochement pratique et symbolique avec l'univers médical a porté ses fruits. Au sein du petit monde funéraire, les thanatopracteurs sont labellisés avant l'heure et disposent ici d'un puissant vecteur d'autonomisation de leur profession face aux opérateurs funéraires.

Spécialistes de cette « vie après la mort », les thanatopracteurs sont ainsi devenus un corps de métier spécifique au sein du monde funéraire, avec notamment un contrôle autonome de la formation et de la qualification ${ }^{2}$, et donc du marché du travail. Qui plus est, ils ont acquis une légitimité supérieure dans la hiérarchie des métiers funéraires. Les thanatopracteurs incarnent aujourd'hui la face noble du funéraire, alliant expertise médicale dans le traitement du corps et activité de restauration/réparation de la personne défunte. C'est ainsi que des anciens « nettoyeurs » sont aujourd'hui volontiers désignés comme les « artistes » du funéraire. Certes, comme partout dans le secteur funéraire, le marché de l'emploi est caractérisé par un déficit de demande : le recrutement est difficile, le recours à du personnel contractuel mal formé et peu motivé est fréquent. Mais le label privilégié que constitue le diplôme d'État leur confère une attractivité meilleure vis-à-vis de jeunes, y compris de jeunes femmes, positivement orientés vers ce choix professionnel.

\section{Un acteur collectif improbable}

La thanatopraxie est donc une activité spécialisée qui implique aujourd'hui l'acquisition de compétences techniques sanctionnées par un diplôme d'État. Le diplôme d'État et les juridictions qui l'accompagnent participent incontestablement d'une fermeture de la communauté des thanatopracteurs, notamment vis-à-vis des autres intervenants du secteur, soignants et pompes

1 En dépit du fait que certains soignants des chambres mortuaires aient exercé une activité comparable à celles des thanatopracteurs durant plusieurs décennies.

2 La formation est composée d'une partie théorique réalisée et validée par les facultés de médecine, une partie pratique de 100 soins réalisés sous contrôle d'un praticien et validée par un jury professionnel. 
funèbres. Le contrôle du marché du travail via la maitrise de l'accès à la qualification a permis aux thanatopracteurs de conforter leur position sur le marché des services funéraires. Ceux ci sont rares et très recherchés, ce qui leur assure une position privilégiée dans la hiérarchie salariale des agents du secteur. Mais ce ressort de l'autonomisation professionnelle ne saurait en soi suffire à construire une réelle capacité de gouvernement de leur marché.

L'univers des professionnels de la thanatopraxie reconnaît une diversité de structures de travail et d'emploi. De grandes entreprises spécialisées rayonnent à l'échelon du territoire régional, ce qui n'exclut pas la présence de multiples petits indépendants dispersés et travaillant seuls. Le thanatopracteur peut également exercer en qualité de salarié au sein d'une entreprise de pompes funèbres disposant des capacités suffisantes. Mais aucun de ces statuts n'autorise une réelle émancipation de la « tutelle des pompes funèbres ». Au contraire, l'hyperspécialisation a davantage conduit les professionnels à se trouver en situation de dépendance stricte face à leurs prescripteurs (opérateurs funéraires). L'intégration des métiers, notamment ceux des pompes funèbres (conseiller ou directeur de structure) et celui de thanatopracteur, autoriserait à l'évidence un meilleur contrôle du marché. Sur les 700 thanatopracteurs environ que compte le territoire national, la qualité des soins mortuaires apportée aux défunts demeure encore très variable selon les professionnels. La pression du marché (rapidité des soins, concurrence sur les prix ${ }^{1}$ ) est un facteur essentiel de ce phénomène.

À la tutelle des pompes funèbres, les thanatopracteurs ajoutent la faiblesse d'une communauté professionnelle qui éprouve d'importantes difficultés à se construire comme acteur collectif. Entre de grandes entreprises reliées à des intérêts concurrents sur le marché et une masse de petits indépendants atomisés, on peut ainsi aisément concevoir les difficultés du syndicalisme professionnel à s'unifier pour se constituer comme interlocuteur légitime vis-à-vis de l'État. Les logiques concurrentielles prennent le pas sur l'enjeu politique d'élaboration d'une régulation collective de la profession, ne serait-ce que pour garantir son autonomie et sa légitimité comme groupe professionnel. En témoigne l'importance des controverses concernant le contrôle de la qualification, alors que celui-ci constitue le vecteur essentiel de structuration professionnelle. L'actualité du diplôme national de thanatopraxie est exclusivement animée par la querelle entre les micropuissances patronales et leurs structures de formation concurrentes. Si elle questionne à l'évidence le rôle des pouvoirs publics comme « tiers garant », elle interroge plus fondamentalement la capacité d'auto-organisation de ce groupe professionnel. Celle-ci ne dépend-elle pas davantage de sa capacité à tracer une limite précise entre les professions reconnues et les autres, entre les clercs et les profanes, afin d'empêcher - ou non - l'intrusion de ceux-ci dans les affaires de ceux-là ? L'enjeu constitué par la nature du savoir en

1 Des praticiens n'hésitent pas à se lancer dans une guerre des prix en proposant trois ou quatre soins en $1 \mathrm{~h} 30$. 
œuvre n'est pas la revendication de son monopole mais bien son organisation interne (Montjardet, 1987).

\section{口 Conclusion}

Dans cet article, nous nous sommes penchés sur les dynamiques professionnelles dans le secteur des services aux défunts, en prenant en compte plusieurs segments professionnels offrant autant d'éclairages sur l'évolution du champ de ce type de service. Nous avons tout d'abord observé qu'en amont, du côté de l'hôpital et de son interface avec les opérateurs funéraires, cohabitent des catégories d'agent d'exécution œuvrant en chambre mortuaire et dans les entreprises de pompes funèbres. Ces agents sont conduits à interagir au quotidien et à se coordonner. Or, cette interface, entre secteur public et secteur privé, est à la fois au cœur d'enjeux économiques importants et de nouvelles stratégies hospitalières conduisant à réinterroger la division du travail et l'articulation entre service public et offre marchande, soignants et pompes funèbres. Portant ensuite notre attention sur le personnage emblématique du conseiller funéraire, dont nous avons décliné les registres de compétence, nous avons montré qu'il conjugue le traitement du corps, la gestion administrative et la ritualité funéraire au travers de la coordination d'une pluralité d'intervenants. Figure hybride et changeante au cœur des réseaux notables et religieux de la communauté locale, ce conseiller technico-commercial est de plus en plus investi d'un rôle « d'officiant» dans la production des «biens symboliques » jusqu'alors monopolisée par les acteurs religieux. Troisième figure professionnelle de ce parcours dans l'univers des spécialistes des services au défunt, le thanatopracteur traduit un cheminement inverse, celui d'une surspécialisation technique. S'accrochant au monde paramédical en tant que spécialiste du traitement biologique du cadavre, il développe son autonomie jusqu'à constituer une sous-communauté professionnelle, plus forte d'une qualification établie et simultanément plus exposée au repli segmentaire.

Ces éclairages successifs témoignent des déplacements qui s'opèrent au sein du système des professions intervenant tout au long de la chaîne des soins au défunt : reconstruction des formes de division et de coordination du travail ; redéfinition des relations et de la nature des échanges ; recomposition des territoires de compétence professionnelle et compétition entre professionnels; émergence de nouvelles formes de cohabitation entre métiers (soins, conseil, accompagnement...), relevant du public et du privé. Ces mouvements nous rappellent que les frontières de compétences sont instables et en perpétuelle redéfinition, objet de dynamiques de professionnalisation très diverses (Abott, 1988). Il est fort à parier que les transformations observées ne sont pas plus définitives que les agencements prévalant dans le passé, d'autant plus que des stratégies d'entreprise et de professionnalisation concurrentes sont à l'œuvre. 


\section{Bibliographie}

ABOTT A., (1988), The System of Professions. An Essay on the Division of Expert Labor, Chicago, The University of Chicago Press.

BARRAU A., (1987), « Les services funéraires. Permanences et évolutions », Économie et statistique, 198.

BOISSIN O., TROMPETTE P., (2002), Les services funéraires. Du monopole public au marché concurrentiel, Paris, DARES.

CAROLY S., TROMPETTE P., (2004), La compétence de service comme compétence de coordination et d'orchestration. Autour du conseiller funéraire, $4^{\mathrm{e}}$ Conférence du RC52 de l'Association internationale de sociologie, savoir, travail, organisation, université de Versailles.

LEMONNIER M., (2003), « Profusion des terminologies, variété des définitions : la thanatopraxie, une technique d'embaumement pratique méconnue », in Caroly S., Rocchi V., Trompette P., Vinck D., Les services au défunt : acteurs, territoires de compétence, dynamiques professionnelles, Rapport MiRe-DREES.

MEMMI D., (2003), Faire vivre et laisser mourir, Paris, Éditions La Découverte.

MONTJARDET D., (1987), «Compétences et qualification comme principe d'analyse de l'action policière », Sociologie du travail, 1, 47-58.

PARADEISE C., (1987), « Des savoirs aux compétences : qualification et régulation du marché du travail », Sociologie du travail, XXIX, 1, 35-46.

THOMAS L. V., (1980), Le cadavre. De la biologie à l'anthropologie, Bruxelles, Éditions Complexe.

VAN GENNEP A., (1998), Le folklore français, Paris, Éditions Robert Laffont.

VINCK D., (2003), « Autour de la qualité », in Caroly S., Rocchi V., Trompette P., Vinck D., Les services au défunt. Acteurs, territoires de compétence et dynamiques professionnelles, Rapport MiRe-DREES. 